\title{
Alterations of retinal vessels in patients with sepsis
}

\author{
Jurate Simkiene $^{1} \cdot$ Zivile Pranskuniene $^{2,3} \cdot$ Martynas Patasius $^{4} \cdot$ Jurgis Trumpaitis $^{5} \cdot$ E. Christiaan Boerma $^{6}$. \\ Andrius Pranskunas ${ }^{1}$ (I)
}

Received: 5 August 2019 / Accepted: 3 October 2019 / Published online: 24 October 2019

(c) Springer Nature B.V. 2019

\begin{abstract}
To compare the retinal vasculature of septic patients with age-matched healthy volunteers. This is a single-centre prospective observational study from January 2018 to May 2019 in a third-level ICU. We performed a single fundus imaging using a hand-held digital fundus camera in patients with sepsis or septic shock $(\mathrm{n}=40)$ during the first $24 \mathrm{~h}$ after ICU admission and compared these data with age-matched healthy controls $(n=20)$. Semi-automated image analysis was performed. The average retinal arteriolar and venular caliber were calculated and summarized as the central retinal arteriolar equivalent (CRAE) and central retinal venular equivalent (CRVE). Arteriole:venular ratio (AVR) was defined as the ratio of CRAE:CRVE. The vascular length density of segmented retinal vessels was = defined as the skeletonized vessel area/total area $\times 100 \%$. Median CRAE of septic patients was significantly higher in comparison to healthy controls (165[149-187] vs. 146[142-158] $\mu \mathrm{m}$, $\mathrm{p}=0.002)$. However, median CRVE and AVR of septic patients did not differ with healthy controls (247[223-282] vs. 244 [215-272], $\mathrm{p}=0.396$ and $0.64[0.58-0.74]$ vs. $0.61[0.55-0.68], \mathrm{p}=0.145$ ) respectively. Patients with sepsis showed a significant decrease in retinal vascular length density compared with healthy subjects $(\mathrm{p}<0.001)$. Retinal observation using a hand-held fundus imaging device showed signs of arteriolar vasodilation with decreased vascular density in septic patients in comparison to healthy controls.
\end{abstract}

Keywords Retinal vascular density $\cdot$ Retinal vascular diameters $\cdot$ Fundus imaging $\cdot$ Intensive care unit $\cdot$ Sepsis

Andrius Pranskunas

andrius.pranskunas@1smuni.lt

1 Department of Intensive Care Medicine, Lithuanian University of Health Sciences, Eiveniu str. 2, 50009 Kaunas, Lithuania

2 Department of Drug Technology and Social Pharmacy, Lithuanian University of Health Sciences, Eiveniu str. 2, 50009 Kaunas, Lithuania

3 Institute of Pharmaceutical Technologies, Lithuanian University of Health Sciences, Eiveniu str. 2, 50009 Kaunas, Lithuania

4 Department of Applied Informatics, Kaunas University of Technology, K. Donelaicio St. 73, 44249 Kaunas, Lithuania

5 Department of Ophthalmology, Lithuanian University of Health Sciences, Eiveniu str. 2, 50009 Kaunas, Lithuania

6 Department of Intensive Care Medicine, Medical Center Leeuwarden, Henri Dunantweg 2, Leeuwarden 8901 BR, The Netherlands

\section{Introduction}

The retina is a window where the microvascular profile can be imaged directly at the bedside in a non-invasive way. Studies already showed that major non-eye diseases, such as diabetes, hypertension, coronary heart disease and stroke, display changes in the retinal vasculature and can even be diagnosed by these alterations. Studies on arterial hypertension and diabetes demonstrated a correlation between retinal arteriolar diameter or, retinal arteriolar:venular ratio (AVR) and arterial blood pressure $[1,2]$ Wide retinal venules and narrow arterioles are associated with increased risk of mortality and ischemic stroke [3].

Sepsis is associated with high morbidity and mortality, even with optimal treatment [4]. The microcirculation is the primary vascular compartment of alterations in sepsis. For practical purposes the sublingual location is the mostly investigated in the clinical setting, using hand-held microscopes [5]. Microcirculatory alterations in sepsis, such as a decrease in microvascular density and flow, occur early and its severity and persistence are associated with organ failure 
and mortality [5-7]. These alterations can be observed in all other studied organs, including the brain [8] and conjunctiva [9]. A study using retinal angiography demonstrated that prolonged retinal arterial filling time is associated with retinal angiopathies, lower cardiac index, lower levels of inflammatory markers and higher intraocular pressure [10]. However, features of retinal vessels calibers and density during sepsis are not fully characterized.

Observation of the retinal fundus with a dedicated handheld device is non-invasive and can be performed easily and fast in critically ill patients. The aim of our study was to compare the retinal vasculature between septic patients and age-matched healthy volunteers.

\section{Materials and methods}

\subsection{Setting and patients}

This single-centre prospective observational study was performed between January 2018 and May 2019 in 18-bed mixed ICU in a tertiary teaching hospital (The Hospital of Lithuanian University of Health Sciences). The study was approved by Kaunas Regional Biomedical Research Ethics Committee (Ethics approval number No. 2018/BE-2-18) and performed in compliance with the Helsinki Declaration. Written informed consent was obtained from the patients or next-of-kin, consistent with applicable laws. Written informed consent in the control group was obtained from the healthy individuals.

We included patients with sepsis or septic shock the first $24 \mathrm{~h}$ after ICU admission. Exclusion criteria were psychiatric disorders, brain diseases, chronic alcoholism, autoimmune rheumatic and various ophthalmological diseases, such as glaucoma, age-related macular degeneration, diabetic retinopathy, cataract. Hemodynamic variables were measured with a transpulmonary thermodilution device (PiCCO, Pulsion Medical systems, Germany). Intraocular pressure (IOP) was measured by impression tonometry (Shiotz tonometer, Riester, Germany).

The control group consisted of twenty age-matched healthy volunteers with no reported ocular pathology, diabetes, non-controlled hypertension or any clinical eye-related complaint.

\subsection{Fundus imaging and analysis}

Twenty minutes after instillation of tropicamide (1\%) color optic disc photographs were taken in mydriasis, using an Optomed Aurora (Optomed Oy, Finland) digital fundus camera (field of view $50^{\circ}$ ). Measurements of vessel diameters were performed in the right eye. When the right eye was not feasible, the assessment was carried out in the left eye.
Image analysis was performed using validated automatic retinal image analysis (ARIA) software [11].

The calibration factor was determined by measuring the distance between the center of the macula to the center of the optic disc in a sample of 20 color photographs containing the macula and the optic disc in the same field, using a customized caliper tool in ImageJ software. By assuming that the distance from the center of the optic disc to the center of the macula is $4760 \mu \mathrm{m}$ [12], we calculated the scale factor by the following formula: Scale factor $=4760 \mu \mathrm{m} /$ number of pixels from center of macula to center of optic disc. For this study, the calibration factor was $6.87 \mu \mathrm{m} / \mathrm{px}$.

The calibration values per pixel were entered directly into the ARIA program, which then produced calibrated measurements of diameters and lengths of retinal vessels. At the end of the vessel analysis, ARIA produces a summary table for each patient with mean $( \pm S D)$ diameter and length of each vessel within the analyzed area. Automatically numbered vessels then were classified in arteries and veins [13].

We measured the diameters of the biggest six arterioles and venules coursing through an area of $0.5-1$ disc diameter surrounding the optic disc (zone B). Based on the revised Knudtson-Parr-Hubbard formula, the average retinal arteriolar and venular caliber were calculated and summarized as the central retinal arteriolar equivalent (CRAE) and central retinal venular equivalent (CRVE) [14]. Arteriole:venular ratio was defined as the ratio of CRAE:CRVE.

The average tortuosity of arterioles and venules was measured within the region between 0.5 and 2.0 disc diameters away from the disc margin (zone C).

The manual segmentation was performed using "Vampire" software (The Manual vessel segmentation tool is made available by the VAMPIRE group at vampire.computing.dundee.ac.uk.). The density of manually segmented retinal vessels was calculated using ImageJ software: vascular length density $=$ skeletonized vessel area/total area $\times 100 \%$.

\subsection{Data collections}

The following data were recorded: general characteristics, routine laboratory results, ventilator settings, and severity of illness according to Acute Physiology and Chronic Health Evaluation (APACHE) II and Sequential Organ Failure Assessment (SOFA) scores.

\subsection{Statistics}

Primary outcomes were CRAE and CRVE. Considering similar conducted studies, and detected means and standard deviations in pilot observations in both groups we estimated that a sample size of 40 patients and 20 healthy control should be sufficient (power $80 \%$, alpha risk 5\%). Data were analyzed with Statistical Package for Social Sciences 
(SPSS 22 for Windows, Chicago, IL, USA). Data are presented as the median [interquartile range, IQR] and analyzed with non-parametric tests. Between groups differences were tested with a Mann-Whitney $U$ test. Correlations between changes in retinal vascular parameters and systemic hemodynamic parameters were tested with a Spearman correlations test. A $p$ value of $<0.05$ was considered significant.

\section{Results}

\subsection{General data}

We investigated 49 patients, but due to poor retinal image quality only 40 patients were included. Baseline characteristics of included patients are shown in Table 1. Out of 40 patients, 36 (90\%) were in septic shock, $40(100 \%)$ required mechanical ventilation. Median APACHE II and SOFA scores were 16[11-19] and 9[6-11], respectively. Mean arterial pressure (MAP) and cardiac index (CI) were $74[65-81] \mathrm{mmHg}$ and $3.0[2.2-4.0] \mathrm{L} / \mathrm{min} / \mathrm{m}^{2}$, respectively. Median norepinephrine (NE) dose was 0.24[0.09-0.32] mcg/ $\mathrm{kg} / \mathrm{min}$.

\subsection{Retinal vessel calibers and density}

Median CRAE of septic patients was significantly higher in comparison to healthy controls (165[149-187] vs. 146[142-158] $\mu \mathrm{m}, \mathrm{p}=0.002)$. However, median CRVE and AVR of septic patients did not differ with healthy controls $(247[223-282]$ vs. $244[215-272], p=0.396$ and $0.64[0.58-0.74]$ vs. $0.61[0.55-0.68], p=0.145$ ) respectively. Patients with sepsis showed a significant decrease in retinal vascular length density compared with healthy subjects $(\mathrm{p}=<0.001$, Table 2, Fig. 1).

To evaluate the possible effects of a history of hypertension on the retinal vessels, we divided septic patients into two subgroups: with and without a history of hypertension (Table 3). We found that patients with a history of hypertension were significantly older, had a higher Apache II score, lactates and noradrenaline dose but there was no difference in retinal vascular diameters.

\subsection{Correlations between retinal vessel parameters and systemic hemodynamic parameters}

We found a significant correlation between CRVE and lactate $(r=-0.442, p=0.005)$, AVR and lactate $(r=0.459$, $\mathrm{p}=0.003)$, between arterial tortuosity and diastolic blood pressure $(\mathrm{r}=-0.351, \mathrm{p}=0.036)$, between vascular length density and lactate $(r=-0.317, p=0.047)$, vascular length density and CI $(r=0.540, p=0.014)$. However, we did not find a relationship between MAP, heart rate, and retinal
Table 1 General data

\begin{tabular}{ll}
\hline & Septic patients $(\mathrm{n}=40)$ \\
\hline Age (years) & $67[55-76]$ \\
Gender (n male; $\mathrm{n}$ female) & $25 ; 15$ \\
Severity of sepsis & \\
Sepsis & 4 \\
Septic shock & 36 \\
Source of sepsis & \\
Pneumonia & 9 \\
Urinary tract infection & 3 \\
Skin and soft tissue infection & 1 \\
Peritonitis & 16 \\
Other infection & 11 \\
Time after admission $(\mathrm{hours})$ & $12[7-18]$ \\
APACHE II score & $16[11-19]$ \\
SOFA score & $9[6-11]$ \\
Temperature $\left({ }^{\circ} \mathrm{C}\right)$ & $37.1[36.3-38.1]$ \\
Heart rate $(\mathrm{bpm})$ & $96[81-129]$ \\
Mean arterial $\mathrm{pressure}(\mathrm{mm} \mathrm{Hg})$ & $74[65-81]$ \\
Intraocular $\mathrm{pressure}(\mathrm{mmHg})$ & $17.3[17.3-17.5]$ \\
Cardiac index $\left(\mathrm{L} / \mathrm{min} / \mathrm{m}^{2}\right)$ & $3.0[2.2-4.0]$ \\
Creatinine $(\mathrm{umol} / \mathrm{L})$ & $177[121-288]$ \\
Bilirubin $(\mathrm{mmol} / \mathrm{L})$ & $17[10-31]$ \\
Platelet count $\left(\times 10^{9} / \mathrm{L}\right)$ & $219[115-302]$ \\
Leukocyte count $\left(\times 10^{9} / \mathrm{L}\right)$ & $14[8-18]$ \\
CRP $(\mathrm{mg} / \mathrm{L})$ & $217[151-282]$ \\
Lactate $(\mathrm{mmol} / \mathrm{L})$ & $2[1-3]$ \\
Norepinephrine $(\mathrm{n} ; \mu \mathrm{g} / \mathrm{kg} / \mathrm{min})$ & $36 ; 0.24[0.09-0.32]$ \\
Adrenaline $(\mathrm{n} ; \mu \mathrm{g} / \mathrm{kg} / \mathrm{min})$ & $3 ; 0.06[0.06-0.06]$ \\
Dobutamine $(\mathrm{n} ; \mu \mathrm{g} / \mathrm{kg} / \mathrm{min})$ & $1 ; 6[6-6]$ \\
ScvO ${ }_{2}(\%)$ & $75[71-78]$ \\
\hline & \\
\hline
\end{tabular}

All data are represented as median [IQR] unless stated otherwise $A P A C H E$ acute physiology and chronic health evaluation, SOFA sequential organ failure assessment, $\mathrm{CRP}$ C-reactive protein, $\mathrm{SvO}$ central venous oxygen saturation

vascular parameters. The correlation between noradrenaline dose and CRAE was not significant: $\mathrm{r}=-0.29, \mathrm{p}=0.092$.

\section{Discussion}

To our knowledge we are the first to describe retinal vascular parameters in patients with sepsis using a hand-held fundus camera. We found that patients with sepsis had increased retinal arteriolar calibers compared with controls. In a previous publication the authors reported that angiography-derived shorter retinal arterial filling times in septic patients were associated with a higher cardiac index and higher levels of inflammatory markers [10]. However, the authors didn't publish retinal arteriolar diameters, which could be measured 
Table 2 Retinal vessel calibers and density in septic patients and healthy volunteers

\begin{tabular}{llll}
\hline Retinal vascular parameters & $\begin{array}{l}\text { Septic patients } \\
(\mathrm{n}=40)\end{array}$ & $\begin{array}{l}\text { Healthy volunteers } \\
(\mathrm{n}=20)\end{array}$ & $P$ value \\
\hline CRAE $(\mu \mathrm{m})$ & $165[149-187]$ & $146[142-158]$ & 0.002 \\
CRVE $(\mu \mathrm{m})$ & $247[223-282]$ & $244[215-272]$ & 0.396 \\
AVR & $0.64[0.58-0.74]$ & $0.61[0.55-0.68]$ & 0.145 \\
Arteriolar tortuosity & $1.06[1.04-1.08]$ & $1.06[1.03-1.07]$ & 0.501 \\
Venular tortuosity & $1.03[1.02-1.05]$ & $1.02[1.02-1.03]$ & 0.005 \\
Vascular length density $(\%)$ & $0.51[0.43-0.56]$ & $0.64[0.60-0.69]$ & $<0.001$ \\
\hline
\end{tabular}

All data are represented as median [IQR] unless stated otherwise

$C R A E$ central retinal arteriolar equivalent, $C R V E$ central retinal venular equivalent, $A V R$ arteriolar:venular ratio
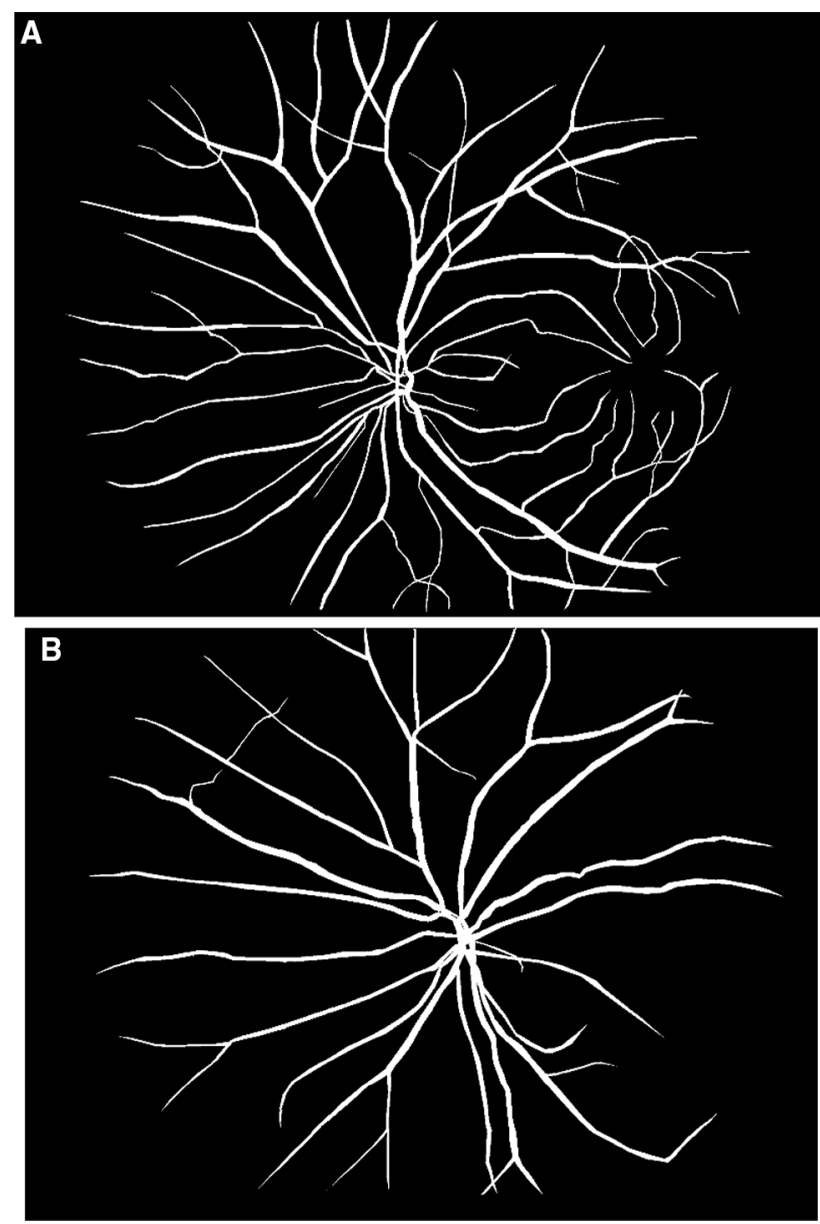

Fig. 1 Manually segmented retinal vascular networks: a healthy volunteer (vascular length density $0.68 \%$ ), b septic patient (vascular length density $0.43 \%$ )

non-invasively at the bedside. Analogous to this publication, median CI in our study was high, suggesting hyperdynamic shock, reflected by retinal arteriolar vasodilation.

Studies with non-critical ill patients or healthy subjects showed that decreased retinal arteriolar diameters predicts coronary heart disease events as well as mortality [3, 15,
16]. Wider venular diameter appears to predict both coronary heart disease and stroke, including mortality from these causes $[3,17]$. There is also a connection between a lower arteriole:venule diameter ratio and increased blood pressure [18], as well as mortality [19]. In other words: in case of atherosclerotic disease states smaller retinal parameters correlate with the occlusive nature of the disease, whereas in sepsis increased retinal parameters reflect the vasodilatory state of the disease.

We found no significant correlation between mean arterial pressure, heart rate, noradrenaline dose and retinal vascular diameters. This may be explained by the concept of loss of vascular tone during septic shock. The dose of vasopressor to maintain vascular tone is individualized to each patient. In addition is seems relevant that the retina is situated close to the brain. Crippa and colleagues [20] observed that cerebral autoregulation was altered in half of the patients with sepsis and that this was associated with the development of sepsis-associated brain dysfunction. Impaired cerebral autoregulation means that mean arterial blood pressure directly correlates with cerebral artery blood flow, where Pearson's correlation coefficient is $r>0.3$. Thus, to maintain cerebral blood flow may require higher blood pressure. In order for the retinal and cerebral circulations to maintain cerebral blood flow over a range of systemic blood pressures, their vascular resistance has to be altered accordingly, when intraocular pressure and intracranial pressure are not elevated [21]. In our study septic patients had close to normal intraocular pressure.

We detected a significant correlation between retinal vessel density parameter and cardiac index. Previous studies demonstrated that changes in the sublingual microcirculation are not related to systemic hemodynamic changes $[22,23]$. This discrepancy can not only be explained by the difference in localization, but also by the difference in the diameter of the blood vessels. Alterations during sepsis using hand-held microscopes were mostly evaluated in vessels smaller than 20 microns, representing mostly capillaries. Due to the difference in magnification during 
Table 3 Comparison of variables in septic patients with and without a history of hypertension

\begin{tabular}{lllc}
\hline & $\begin{array}{l}\text { History of hypertension } \\
(\mathrm{n}=22)\end{array}$ & $\begin{array}{l}\text { No history of hypertension } \\
(\mathrm{n}=18)\end{array}$ & $\mathrm{p}$ \\
\hline Age (years) & $76[67-80]$ & $54[41-63]$ & $<0.001$ \\
APACHE II score & $18[13-25]$ & $13[9-16]$ & 0.007 \\
SOFA score & $10[8-11]$ & $8[6-10]$ & 0.09 \\
Heart rate $(\mathrm{bpm})$ & $108[80-130]$ & $90[80-115]$ & 0.384 \\
Mean arterial pressure $(\mathrm{mm} \mathrm{Hg})$ & $74[66-81]$ & $73[64-82]$ & 0.892 \\
Lactate $(\mathrm{mmol} / \mathrm{L})$ & $1.8[1.3-4.4]$ & $1.4[0.8-1.8]$ & 0.027 \\
Norepinephrine $(\mathrm{n} ; \mu \mathrm{g} / \mathrm{kg} / \mathrm{min})$ & $0.31[0.20-0.36]$ & $0.16[0.08-0.27]$ & 0.038 \\
CRAE $(\mu \mathrm{m})$ & $162[150-179]$ & $180[147-193]$ & 0.202 \\
CRVE $(\mu \mathrm{m})$ & $241[227-278]$ & $266[219-297]$ & 0.497 \\
AVR & $0.65[0.60-0.70]$ & $0.60[0.60-0.80]$ & 0.758 \\
Vascular length density $(\%)$ & $0.47[0.41-0.54]$ & $0.54[0.48-0.64]$ & 0.053 \\
\hline
\end{tabular}

All data are represented as median [IQR]

APACHE acute physiology and chronic health evaluation, SOFA sequential organ failure assessment, CRAE central retinal arteriolar equivalent, $C R V E$ central retinal venular equivalent, $A V R$ arteriolar:venular ratio retinal fundoscopy not many capillaries are visible. In this way, a larger diameter blood vessel can be associated with systemic hemodynamics.

Another finding in our study was that patients with sepsis showed a significant decrease in retinal vascular length density as compared with healthy subjects. Animal studies already showed that perfused vessel density during sepsis is decreased in the ocular conjunctiva $[9,24]$ and brain [8]. In this case a retinal change is also expected. Blood flow of the bulbar conjunctiva and retina may to some extend `represent' the cerebral blood flow, since the ophthalmic artery originates from the internal carotid artery and supplies blood to both retina and bulbar conjunctiva.

There are a few limitations to our study. The study is singlecenter and population is small, but we compared data with agematched volunteers group and our results are observational and hypothesis-generating. Secondly, vessel length density data are dependent on the quality of the retinal photographs and disk centering. However, we made a surplus of images and excluded non gradable photographs. Third, in our study 22 of 40 patients had a history of hypertension. Primary uncontrolled hypertension is characterized by the smaller number of arterioles and capillaries (known as rarefaction) and narrowed retinal arteriolar diameter $[18,25,26]$. However, we included patients with history of controlled hypertension and we did not find a significant difference in arteriolar diameter between patients with a history of hypertension and those without a history of hypertension. Although subgroup of patients with a history of hypertension tended to be less vascular density, this is likely to be associated with septic shock. These patients were more severe (a higher Apache II score), had more lactates and a higher dose of vasopressor. Furthermore, studies have suggested that treatment with antihypertensive medications may reverse or even prevent microvascular rarefaction in patients with controlled hypertension [27, 28].

\section{Conclusions}

Retinal observation using hand-held fundus imaging device showed retinal signs of arteriolar vasodilation with decreased vascular density in septic patients. Retinal observation using hand-held fundus imaging device could be a feasible monitoring technique for the ICU. Further studies are needed to determine the relationship of retinal vessels changes with mortality, systemic hemodynamics and cerebral blood flow.

Author contributions AP, JS was responsible for data acquisition, fundus imaging, image blinding and analysis and design of the study; wrote the first draft; ZP performed image analysis, wrote some aspects of the first draft; JT performed image analysis; MP critically revised the manuscript and participated in data interpretation; $\mathrm{CB}$ contributed to the design of the study and wrote the final manuscript. All authors read and approved the final manuscript.

Funding This research was funded by a Grant (No. S-MIP-17-16) from the Research Council of Lithuania.

\section{Compliance with ethical standards}

Conflict of interest The authors declare that they have no conflict of interest.

\section{References}

1. Ikram MK, de Jong FJ, Vingerling JR, Witteman JC, Hofman A, Breteler MM, de Jong PT. Are retinal arteriolar or venular diameters associated with markers for cardiovascular disorders? The Rotterdam Study. Invest Ophthalmol Vis Sci. 2004;45:2129-34. 
2. von Hanno T, Bertelsen G, Sjolie AK, Mathiesen EB. Retinal vascular calibres are significantly associated with cardiovascular risk factors: the Tromso Eye Study. Acta Ophthalmol. 2014;92:40-6. https://doi.org/10.1111/aos.12102.

3. Seidelmann SB, Claggett B, Bravo PE, et al. Retinal vessel calibers in predicting long-term cardiovascular outcomes: the atherosclerosis risk in communities study. Circulation. 2016;134:1328-38.

4. Hajj J, Blaine N, Salavaci J, Jacoby D. The "centrality of sepsis": a review on incidence, mortality, and cost of care. Healthcare (Basel). 2018. https://doi.org/10.3390/healthcare6030090.

5. De Backer D, Creteur J, Preiser JC, Dubois MJ, Vincent JL. Microvascular blood flow is altered in patients with sepsis. Am J Respir Crit Care Med. 2002;166:98-104.

6. De Backer D, Donadello K, Sakr Y, Ospina-Tascon G, Salgado D, Scolletta S, Vincent JL. Microcirculatory alterations in patients with severe sepsis: impact of time of assessment and relationship with outcome. Crit Care Med. 2013;41:791-9. https://doi. org/10.1097/CCM.0b013e3182742e8b.

7. Trzeciak S, McCoy JV, Phillip Dellinger R, et al. Early increases in microcirculatory perfusion during protocol-directed resuscitation are associated with reduced multi-organ failure at $24 \mathrm{~h}$ in patients with sepsis. Intensive Care Med. 2008;34:2210-7. https ://doi.org/10.1007/s00134-008-1193-6.

8. Taccone FS, Su F, De Deyne C, et al. Sepsis is associated with altered cerebral microcirculation and tissue hypoxia in experimental peritonitis. Crit Care Med. 2014;42:e114-22. https://doi. org/10.1097/CCM.0b013e3182a641b8.

9. Pranskunas A, Pilvinis V, Dambrauskas Z, et al. Early course of microcirculatory perfusion in eye and digestive tract during hypodynamic sepsis. Crit Care. 2012;16:R83. https://doi.org/10.1186/ cc11341.

10. Erikson K, Liisanantti JH, Hautala N, et al. Retinal arterial blood flow and retinal changes in patients with sepsis: preliminary study using fluorescein angiography. Crit Care. 2017. https://doi. org/10.1186/s13054-017-1676-3

11. Bankhead P, Scholfield CN, McGeown JG, Curtis TM. Fast retinal vessel detection and measurement using wavelets and edge location refinement. PLoS ONE. 2012;7:e32435. https://doi. org/10.1371/journal.pone.0032435.

12. Jonas RA, Wang YX, Yang H, Li JJ, Xu L, Panda-Jonas S, Jonas JB. Optic disc-fovea distance, axial length and parapapillary zones. The Beijing Eye Study 2011. PLoS One. 2015;10:e0138701. https://doi.org/10.1371/journal.pone.0138701.

13. Pilat Anastasia V, Proudlock Frank A, McLean Rebecca J, Lawden Mark C, Gottlob Irene. Morphology of retinal vessels in patients with optic nerve head drusen and optic disc edema. Investig Ophthalmol Vis Sci. 2014;55:3484-90.

14. Knudtson MD, Lee KE, Hubbard LD, Wong TY, Klein R, Klein BE. Revised formulas for summarizing retinal vessel diameters. Curr Eye Res. 2003;27:143-9.

15. Wang JJ, Liew G, Klein R, et al. Retinal vessel diameter and cardiovascular mortality: pooled data analysis from two older populations. Eur Heart J. 2007;28:1984-92.

16. McGeechan K, Liew G, Macaskill P, et al. Meta-analysis: retinal vessel caliber and risk for coronary heart disease. Ann Intern Med. 2009;151:404-13.
17. McGeechan K, Liew G, Macaskill P, et al. Prediction of incident stroke events based on retinal vessel caliber: a systematic review and individual-participant meta-analysis. Am J Epidemiol. 2009;170:1323-32. https://doi.org/10.1093/aje/kwp306.

18. Ikram MK, Witteman JC, Vingerling JR, Breteler MM, Hofman A, de Jong PT. Retinal vessel diameters and risk of hypertension: the Rotterdam Study. Hypertension. 2006;47:189-94.

19. Wong TY, Klein R, Nieto FJ, et al. Retinal microvascular abnormalities and 10-year cardiovascular mortality: a population-based case-control study. Ophthalmology. 2003;110:933-40.

20. Crippa IA, Subira C, Vincent JL, et al. Impaired cerebral autoregulation is associated with brain dysfunction in patients with sepsis. Crit Care. 2018. https://doi.org/10.1186/s13054-018-2258-8.

21. Patton N, Aslam T, Macgillivray T, Pattie A, Deary IJ, Dhillon B. Retinal vascular image analysis as a potential screening tool for cerebrovascular disease: a rationale based on homology between cerebral and retinal microvasculatures. J Anat. 2005;206:319-48.

22. De Backer D, Creteur J, Dubois MJ, Sakr Y, Koch M, Verdant C, Vincent JL. The effects of dobutamine on microcirculatory alterations in patients with septic shock are independent of its systemic effects. Crit Care Med. 2006;34:403-8.

23. Ospina-Tascon G, Neves AP, Occhipinti G, et al. Effects of fluids on microvascular perfusion in patients with severe sepsis. Intensive Care Med. 2010;36:949-55. https://doi.org/10.1007/s0013 4-010-1843-3.

24. Hessler M, Arnemann PH, Zamit F, et al. Monitoring of conjunctival microcirculation reflects sublingual microcirculation in ovine septic and hemorrhagic shock. Shock. 2019;51:479-86. https:// doi.org/10.1097/SHK.0000000000001173.

25. Chew SK, Xie J, Wang JJ. Retinal arteriolar diameter and the prevalence and incidence of hypertension: a systematic review and meta-analysis of their association. Curr Hypertens Rep. 2012;14:144-51. https://doi.org/10.1007/s11906-012-0252-0.

26. Kutschbach P, Wolf S, Sieveking M, Ittel TH, Schulte K, Reim M. Retinal capillary density in patients with arterial hypertension: 2-year follow-up. Graefes Arch Clin Exp Ophthalmol. 1998;236:410-4. https://doi.org/10.1007/s004170050098.

27. Jumar A, Harazny JM, Ott C, Kistner I, Friedrich S, Schmieder RE. Improvement in retinal capillary rarefaction after valsartan treatment in hypertensive patients. J Clin Hypertens (Greenwich). 2016;18:1112-8. https://doi.org/10.1111/jch.12851.

28. Hughes AD, Stanton AV, Jabbar AS, Chapman N, MartinezPerez ME, McG Thom SA. Effect of antihypertensive treatment on retinal microvascular changes in hypertension. J Hypertens. 2008;26:1703-7. https://doi.org/10.1097/HJH.0b013e328304b07 2.

Publisher's Note Springer Nature remains neutral with regard to jurisdictional claims in published maps and institutional affiliations. 